\title{
On structural identifiability analysis of cascaded linear time varying systems in dynamic isotope experiments
}

\author{
Weilu Lin ${ }^{1}$, Mingzhi Huang ${ }^{2}$, Zejian Wang ${ }^{3}, \mathrm{Ju}_{\mathrm{Chu}}^{4}$, Yingping Zhuang ${ }^{3}$, and Siliang \\ Zhang $^{3}$ \\ ${ }^{1}$ East China University of Technology \\ ${ }^{2}$ Affiliation not available \\ ${ }^{3}$ East China University of Science Technology \\ ${ }^{4}$ East China University of Science \& Technology, Institute of Fine Chemicals, State Key \\ Laboratory of Bioreactor Engineering
}

May 5, 2020

\begin{abstract}
A well known issue in metabolic flux analysis with labelling experiments is the structural identifiability analysis. It comes from the fact that some enrichment measurement sets cannot uniquely elucidate all intracellular fluxes. To the best of our knowledge, the structural identifiability analysis of dynamic isotope experiments is not available in the literature. In this work, it is shown that if one measurement plan makes the dynamic isotopic fractions balance equations structurally identifiable then for any arbitrary small time interval the plan also makes the equations structurally identifiable. Based on the fact, in order to resolve the local structural identifiablity problem of the dynamic isotopic fractions balance equations approximated with piecewise affine intracellular fluxes, one should check the local structural identifiablity for the corresponding cascaded linear time invariant system at each sampling point with the approach proposed in our earlier work (Lin \emph\{et al.\}, Math Biosci. 2018; 300:122-129).
\end{abstract}

\section{Hosted file}

Structure_Identifiability_of_Dynamic_13C.pdf available at https://authorea.com/users/299596/ articles/429050-on-structural-identifiability-analysis-of-cascaded-linear-time-varyingsystems-in-dynamic-isotope-experiments 